Mediterránea Ser. Biol. (1989), n.o 11. Pág. 59-76

\title{
NUEVAS CITAS Y COMENTARIOS FAUNÍSTICOS SOBRE LOS QUIRÓPTEROS DE LA COMUNIDAD VALENCIANA
}

\author{
por
JORDI SERRA-COBO ${ }^{1}$ y FÉLIX V. FAUS ${ }^{2}$
}

\section{RESUMEN}

Se aportan 92 citas quiropterológicas nuevas a las 110 menciones bibliográficas existentes hasta la actualidad, ampliándose así a 202 el total de referencias concretas conocidas en la Comunidad Valenciana. Igualmente, se cita por primera vez a Rhinolophus mehelyi, Myotis daubentoni y Myotis emarginatus, incrementándose el listado de especies de murciélagos valencianos a 19. Cabe añadir que 39 de las localidades citadas son nuevas. Se ha calculado la densidad de prospección, obteniéndose un valor de 8.67 citas $/ 1000 \mathrm{~km}^{2}$, resultado todavía bajo para considerar el país aceptablemente prospectado. La especie con mayor frecuencia es Rhinolophus ferrum-equinum seguida de Miniopterus schreibersi, sin embargo a nivel poblacional tiene mayor importancia $M$. schreibersi. Desde un punto de vista biogeográfico, predominan relativamente las especies mediterráneas del norte, seguidas de las mediterráneas del sur y las de origen tropical y subtropical. No obstante, si se analiza la fauna quiropterológica en cuanto a la abundancia, los grupos que presentan mayor biomasa son las mediterráneas del sur y las de origen tropical y subtropical, característica que concuerda con el clima de la región estudiada.

Palabras clave: Quirópteros. Corología. Biogeografía. Comunidad Valenciana.

\section{SUMMARY}

The work adds new records of bats to the 110 already in the literature, therefore enlarging to 202 the number of concrete references known at present in the Comunidad Valenciana. This is the first mentioned evidence of Myotis daubentoni, Myotis emarginatus and Rhinolophus mehelyi, increasing to 19 the list of species of valencian bats. Of all localities visited, 39 were unreported to date. The prospection intensity has reached 8.67 records $/ 1000 \mathrm{~km}^{2}$, value lower than necessary to consider the territory as acceptably investigated. On the other hand, the fauna variety

(1) Departamento de Biología Animal (Vertebrados). Facultad de Biología. Universidad de Barcelona. Avda. Diagonal, 645. Barcelona 08028.

(2) Unidad de Ecología, Departamento de Microbiología. Facultad de CC. Biológicas. Universidad de Valencia. C/. Dr. Moliner, 50 . Burjassot 46100 (Valencia). 
index is 2.19 species/record $/ 1000 \mathrm{~km}^{2}$. The more frequent species is Rhinolophus ferrumequinum, followed by Miniopterus schreibersi, although the later has greater importance at population levels. From biogeographical view, the North Mediterranean species have a relatively higher frequency than South Mediterranean species and others by tropical and subtropical origin. Nevertheless, the later groups have individual numbers greater than the first, according to the climate of the region.

Keys words: Bats. Faunistic. Biogeography. Comunidad Valenciana.

\section{INTRODUCCIÓN}

Antes de abordar el análisis de los nuevos datos faunísticos obtenidos, se ha creído oportuno efectuar un breve resumen histórico que permita una visión de conjunto de lo que ha sido hasta la fecha el estudio de los murciélagos valencianos. A finales del pasado siglo GRAELLS (1897) consideró Nyctalus noctula como una especie de Valencia, aportación de sumo interés pues desde entonces hasta la actualidad sólo se ha dado a conocer una cita concreta, la mencionada por BOSCA (1915). Ya a principios de siglo CABRERA (1904 y 1914) añadió algunos datos faunísticos, si bien la mayoría de ellos eran poco precisos. Por su parte, BOSCA (1915) publicó un trabajo en el que se precisaban las localidades donde habían sido hallados los murciélagos. En él merecen especial atención las citas de $N y$. noctula (dos hembras criando), Pipistrellus savii y Tadarida teniotis. Posteriormente, BAUER (1956) dió a conocer nueva información para la Comunidad Valenciana. Sin embargo, tal y como sucedía en las publicaciones de CABRERA (1904 y 1914), las localidades son en su mayoría poco precisas. Ya en la segunda mitad del siglo actual, el estudio de mayor relevancia fue el realizado por BALCELLS (1967) en el que se analizaban las especies de murciélagos del Levante español. Dicho autor fue el primero en dar una visión faunística global e interpretar biogeográficamente los datos. Además, DONAT (1966) citó varias especies en cavidades de la provincia de Valencia. Recientemente, en la década de los años ochenta, diversos autores han contribuido a ampliar la información de la distribución de los quirópteros valencianos, en especial de las especies cavernícolas (HERREROBORGOÑÓN, 1983 y 1988; SAMARRA y CAROL, 1986; SEVILLA, 1986; FAUS, 1987; HERRERO-BORGOÑÓN y GONZÁLEZ, 1987; SERRA y BALCELLS, 1987; VILLAPLANA, 1988).

El objetivo del presente artículo es dar a conocer las citas inéditas obtenidas durante los últimos años en la Comunidad Valenciana y, a su vez, analizarlas conjuntamente con las ya existentes en la bibliografía. En este sentido, se pretende continuar la labor emprendida por BALCELLS (1967), ampliando los conocimientos corológicos de cada especie e interpretando, en la medida de lo posible, la información desde un punto de vista biogeográfico.

\section{MATERIAL Y MÉTODOS}

En el período comprendido entre los años 1981 y 1987 se efectuaron diversas campañas en las provincias de Valencia, Castellón y Alicante, visitando 
enclaves tanto naturales (simas y cuevas), como artificiales (minas, viviendas humanas y túneles). En el actual estudio se han considerado solamente las localidades en que ha sido detectada la presencia de murciélagos, llegándose a su determinación. Así pues, se han omitido aquellas en las que únicamente se observaron quirópteros volando sin poder precisar la especie, o simplemente había guano en su interior. Se ha considerado como cita la mención de una especie en una localidad, prescindiendo del número de veces que se la haya observado en fechas distintas.

Las citas aportadas tienen dos procedencias distintas: la mayor parte de ellas son fruto de las campañas realizadas por los autores del estudio, mientras que otra parte ha sido cedida por el Dr. E. BALCELLS, la Cátedra de Parasitología de la Facultad de Farmacia de la Universidad de Valencia y J. J. HERRERO-BORGOÑÓN. Los enclaves han sido ordenados alfabéticamente haciendo constar la provincia a la que pertenecen y las especies halladas. En el recuento de las citas se han descartado aquellas que no hacian referencia a una localidad concreta cuando eran de ámbito general. La frecuencia se ha calculado como el tanto por ciento de menciones de una especie respecto al conjunto global de citas, mientras que el índice de diversidad de fauna se ha considerado como el resultado de dividir el número de especies por la densidad de prospección.

Los mapas de distribución pretenden dar una primera visión de la repartición de los murciélagos de la Comunidad Valenciana. En ellos han sido representadas conjuntamente las citas inéditas y las bibliográficas (ver Figs. 1-8).

\section{RESULTADOS}

En 47 del total de las localidades prospectadas se han obtenido citas inéditas para alguna especie de murciélago. Cabe añadir que 39 de dichos enclaves son nuevos, pues si bien algunos de ellos ya habían sido mencionados en anteriores publicaciones, no se llegó a determinar los quirópteros que se refugiaban en su interior. Teniendo en cuenta que el número de localidades citadas en la bibliografía es de 77, se aportan aquí un $52.63 \%$ de menciones nuevas, obteniéndose un total de 116 para la región. A continuación se especifica el catálogo de localidades prospectadas, detallándose solamente las citas quiropterológicas inéditas. Los refugios nuevos vienen indicados con un asterisco, mientras que las iniciales B., H. B. y P. muestran los datos cedidos por E. BALCELLS, J. J. HERRERO-BORGOÑ́N y la Cátedra de Parasitología, respectivamente.

1.- ÁGUILA, sima del (Picassent, Valencia): Myotis blythi, Miniopterus schreibersi

2.- AIGUA, sima de l' (Carcaixent, Valencia) (*): Rhinolophus ferrum-equinum

3.- ALBUFERA (Valencia, Valencia) (*): Pipistrellus pipistrellus, Eptesicus serotinus, Pipistrellus kuhli

4. - BENICALAP (Valencia, Valencia) $\left({ }^{*}\right)$ : P. pipistrellus 


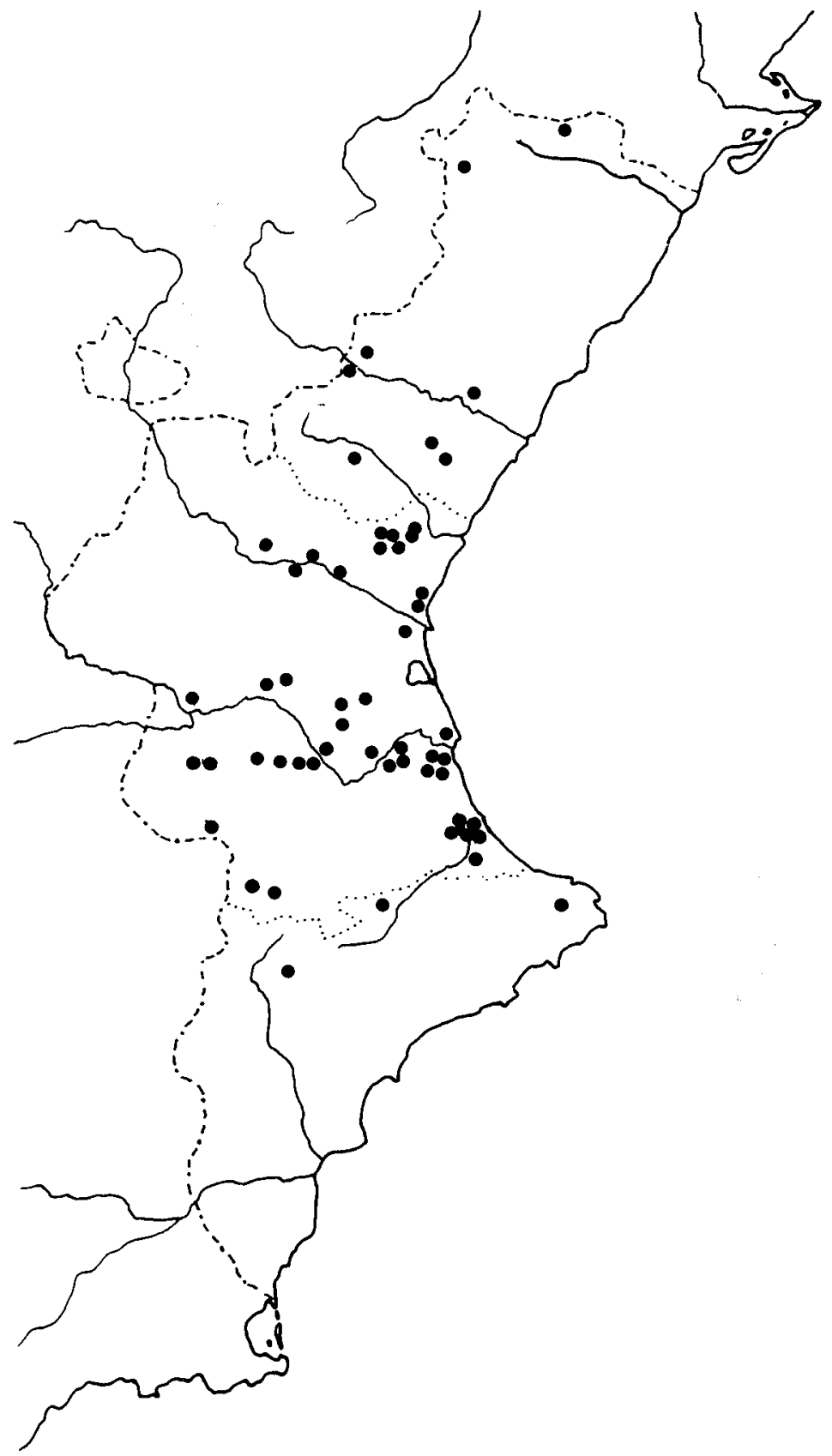

Figura 1.- Rhinolophus ferrum-equinum 


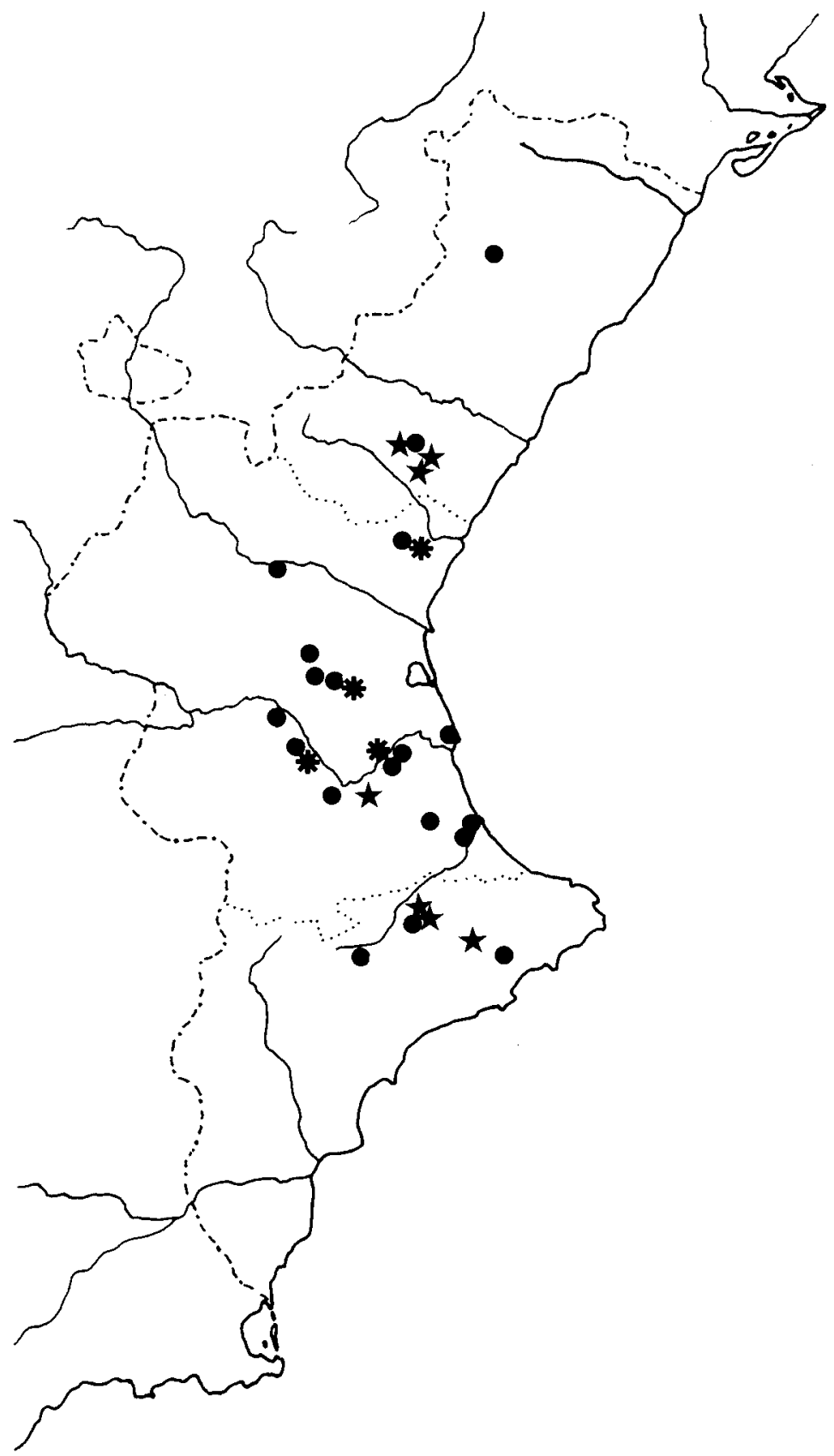

Figura 2.- Rhinolophus euryale

* Rhinolophus mehelyi

$\star$ Rhinolophus hipposideros 


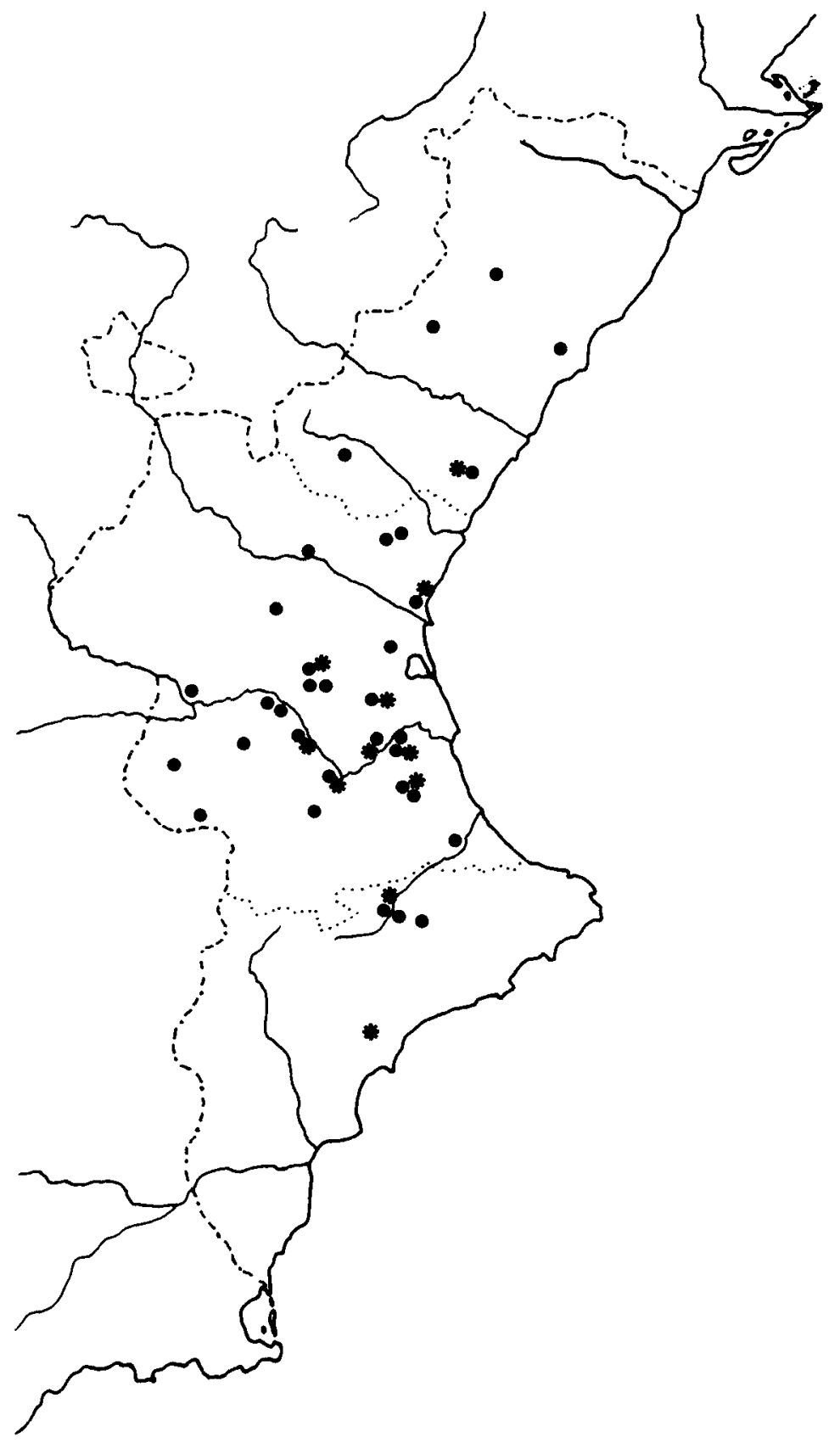

Figura 3.- Miniopterus schreibersi

- Myotis capaccinii 


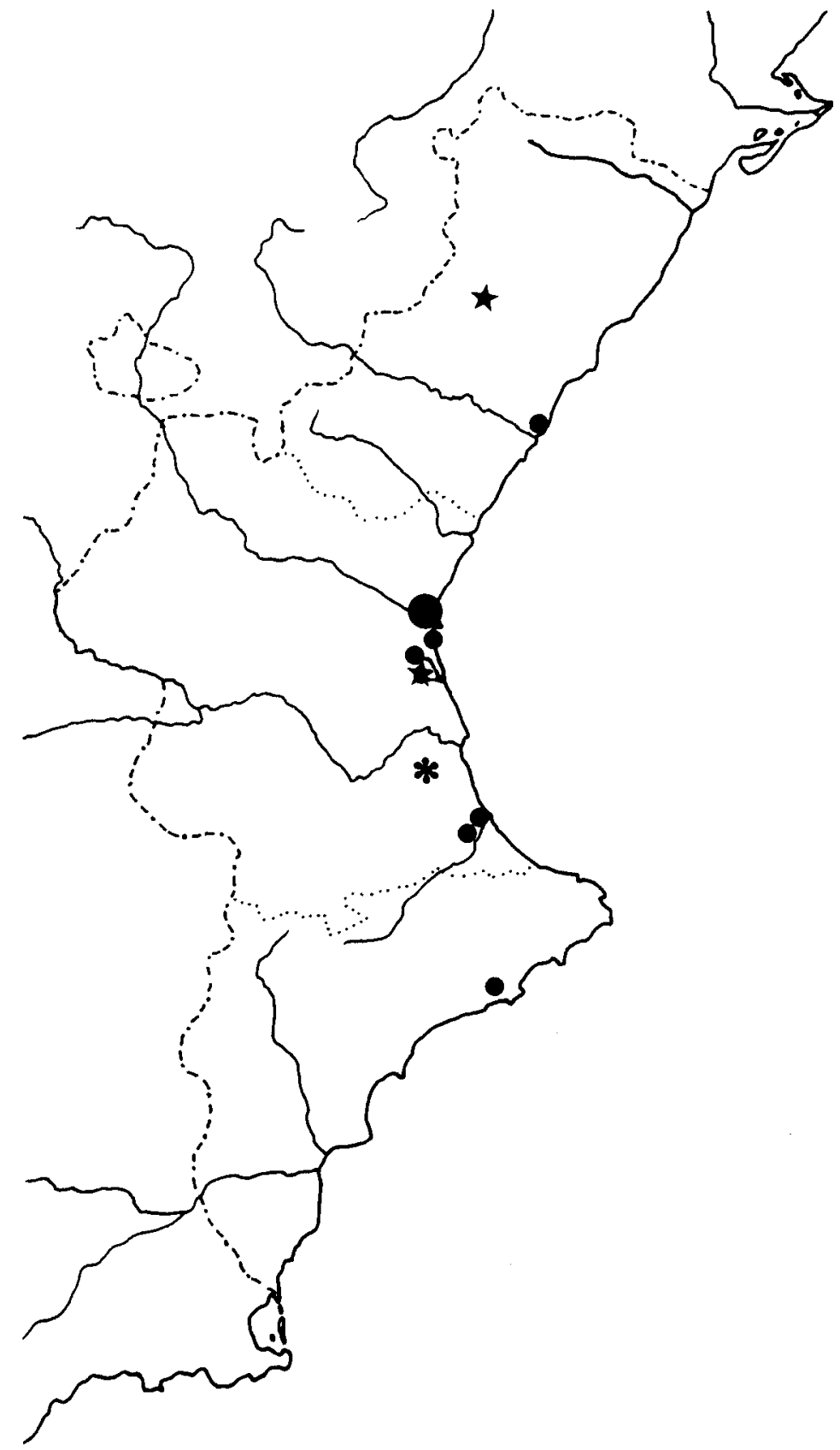

Figura 4.- Pipistrellus pipistrellus $\star$ Pipistrellus kuhli

* Pipistrellus savii 


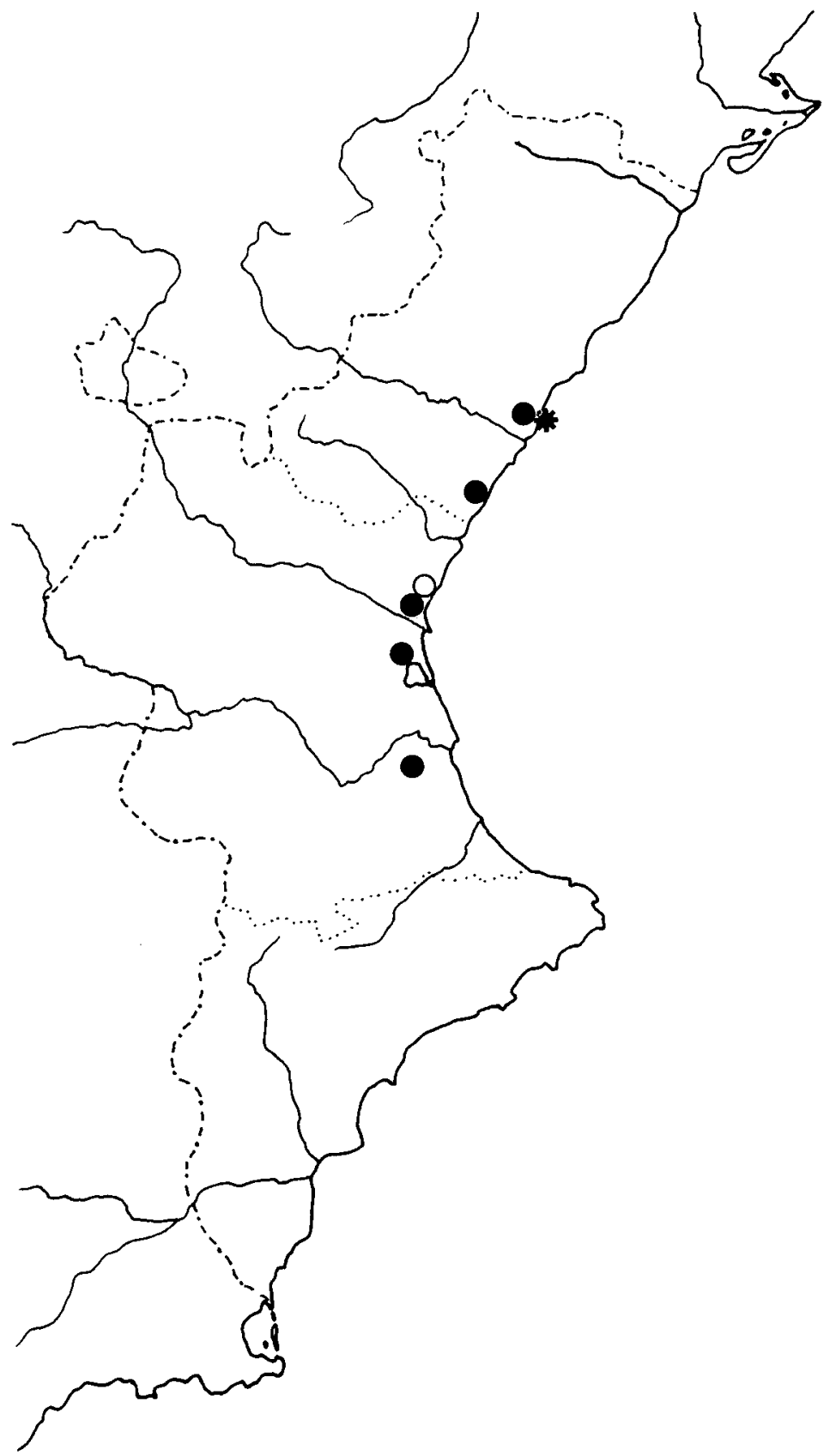

Figura 5.— * Barbastella barbastellus

O Nyctalus noctula

Eptesicus serotinus 


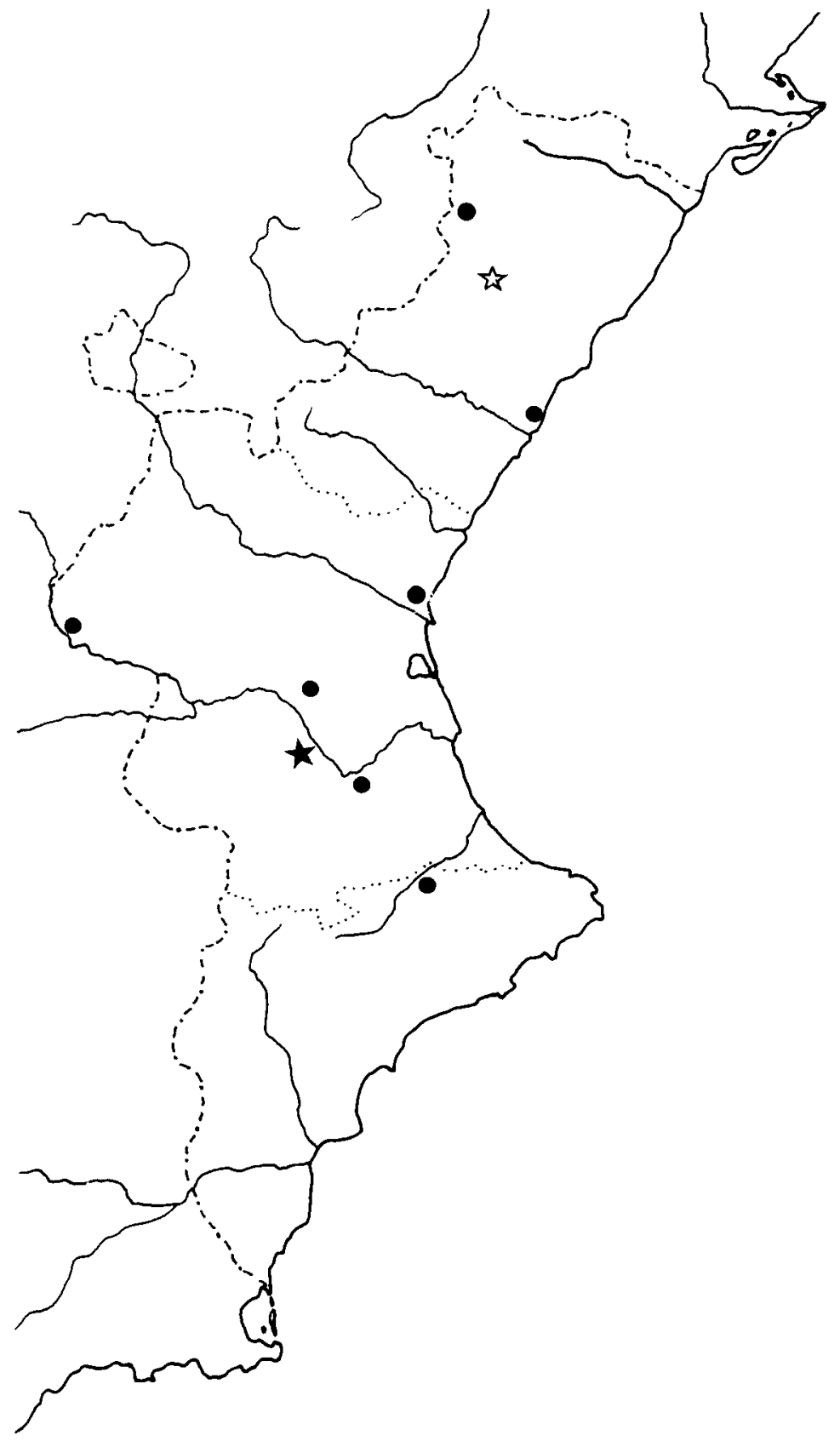

Figura 6.- Myotis nattereri

Myotis daubentoni

$\star$ Myotis emarginatus 


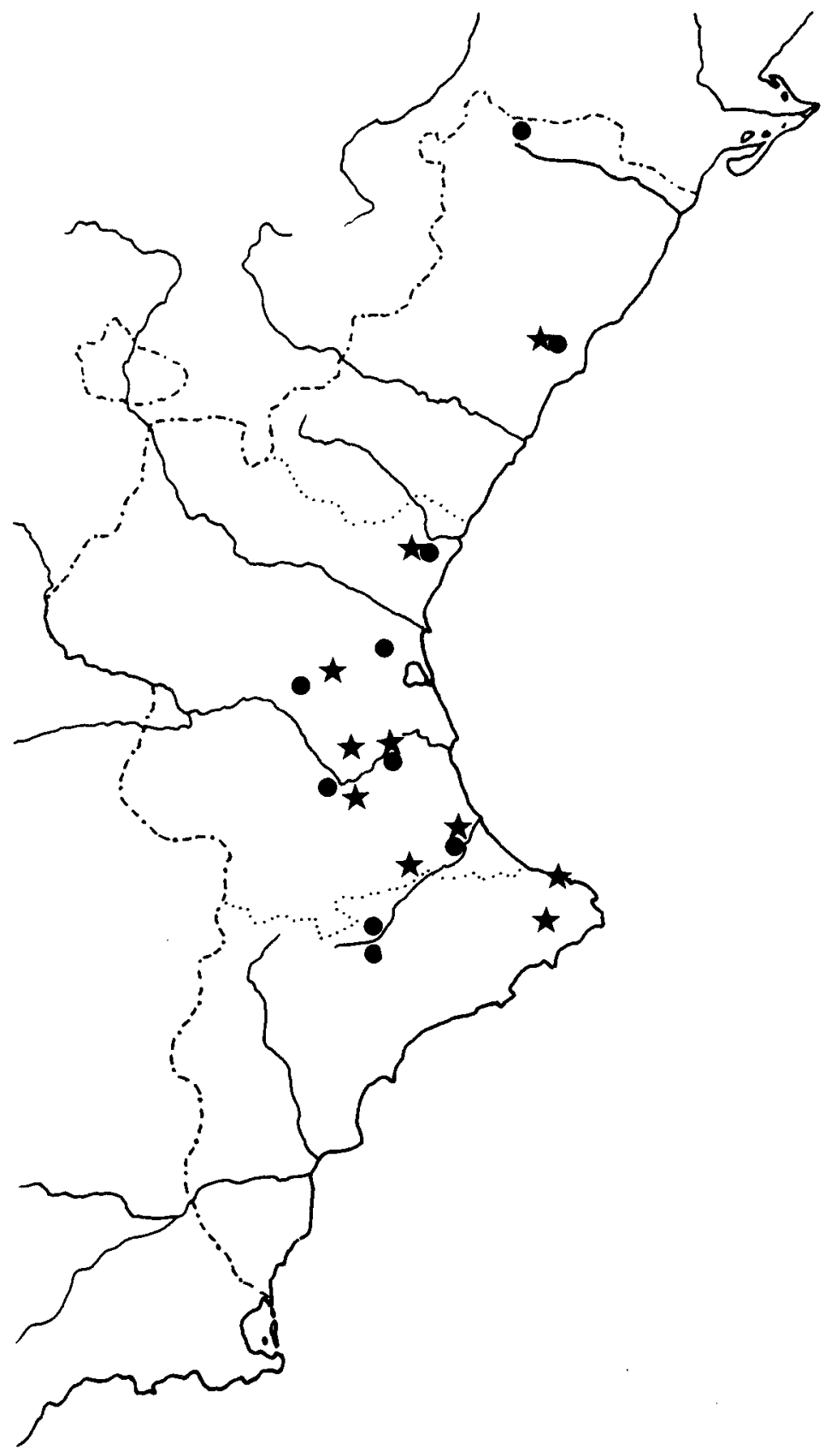

Figura 7.— $\star$ Myotis myotis

- Myotis blythi 


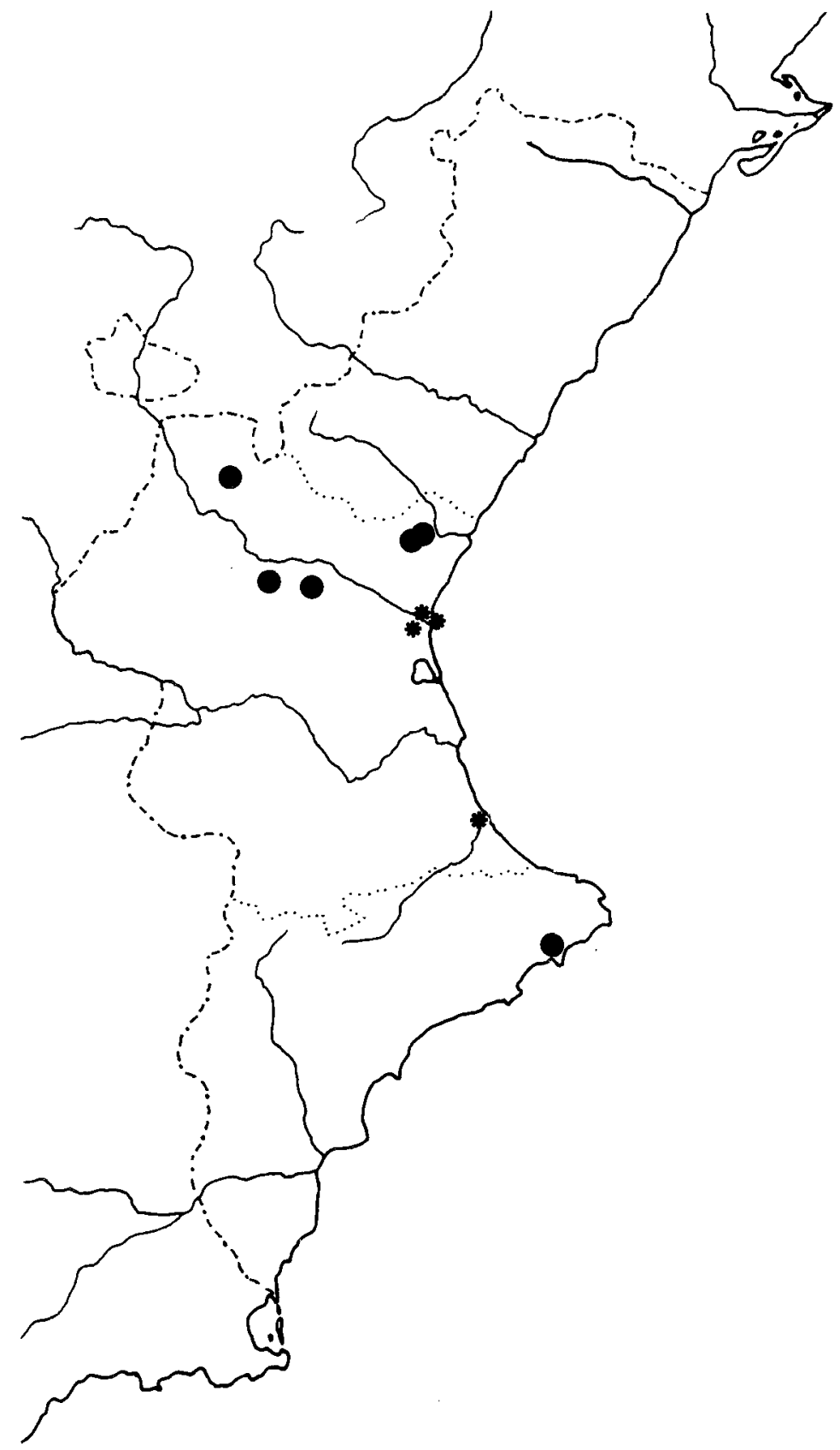

Figura 8.- Plecotus austriacus

* Tadarida teniotis 
5.- CALAVERES, cova de les (Benidoleig, Alicante) $\left({ }^{*}\right)$ : $R h$. ferrum-equinum

6.- CALP, túnel de la carretera de (Calp, Alicante) $\left({ }^{*}\right)$ : Plecotus austriacus

7.- CAMPANAR (Valencia, Valencia) $\left({ }^{*}\right)$ : $P$. pipistrellus (P.)

8.- CAMPILLO, sima del (Tous, Valencia) (*): M. schreibersi, Myotis capaccinii. My. blythi

9.- CANDIL, cueva del (Tous, Valencia): Rhinolophus euryale

10.- CARCALIN, túnel de (Buñol, Valencia) (*): M. schreibersi

11.- COVES, les (Burjassot, Valencia) $\left(^{*}\right)$ : $P$. pipistrellus

12.- GOTINYA, cova (Penáguila, Alicante): $R h$. euryale, Rhinolophus hipposideros

13. - GRAELLES, sima de les (Tous, Valencia) $\left({ }^{*}\right)$ : $M$. schreibersi, My. capaccinii, Rh. ferrum-equinum, Rh. euryale, Rhinolophus mehelyi, Myotis daubentoni

14. - HERMOSA, cueva (Cortes de Pallás, Valencia): M. schreibersi

15.- HIGUERAL, cueva del (Gestalgar, Valencia) $\left(^{*}\right)$ : $R h$. ferrum-equinum (H. B.), Rh. euryale (H. B.)

16. - K-28, sima (Serra, Valencia): M. schreibersi

17.- LLENTISCLE, cova del (Vilamarxant, Valencia) $\left({ }^{*}\right)$ : $R h$. ferrum-equinum

18.- MARAVILAS, cueva de las (Dos Aguas, Valencia) (*): $R h$. ferrum-equinum

19.- MERAVELLES, cova de les (Llombai, Valencia) $\left({ }^{*}\right)$ : Rh. ferrum-equinum, M. schreibersi, My. capaccinii, Rh. euryale, Myotis myotis

20.- MERAVELLES, cova de les (Alzira, Valencia) $\left({ }^{*}\right)$ : $R h$. ferrum-equinum, Rh. eurayale, $M$. schreibersi

21.- MIJARES, aldea de (Buñol, Valencia) $\left({ }^{*}\right)$ : $P$. austriacus (H. B.)

22.- MOLI, sima del (Alberic, Valencia) (*): Rh. ferrum-equinum, M. schreibersi, My. capaccinii, My. myotis

23. - MONEDA, cueva de la (Cotes, Valencia) (*): M. schreibersi, Rh. mehelyi, My. blythi, Rh. euryale, My. capaccinii

24.- MURCIÉLAGOS, cueva de los (Altura, Castellón) $\left({ }^{*}\right)$ : $R h$. ferrum-equinum, $M$. schrebersi

25.- OSCURA, cueva (Atzeneta, Castellón) $\left({ }^{*}\right)$ : $M$. schreibersi, Rh. euryale, Myotis emarginatus, $P$. kuhli

26. - PALOMAS, cueva de las (Millares, Valencia) $\left({ }^{*}\right)$ : Rh. euryale (H. B.), M. schreibersi (H. B.)

27. - PARIDERA, sima de la (Dos Aguas, Valencia) $\left({ }^{*}\right)$ : $R h$. ferrum-equinum

28.- PEN̂A DEL RAYO, cueva del (Tuéjar, Valencia) $\left(^{*}\right)$ : Rh. ferrum-equinum (H. B.) 
29.- PEÑÓN, cueva del (Serra, Valencia) $\left(^{*}\right)$ : Rh. ferrum-equinum (B.)

30.- PEPE LILA, sima de (Bicorb, Valencia) $\left({ }^{*}\right)$ : $R h$. ferrum-equinum, $M$. schreibersi

31.- PLATA, mina de (Serra, Valencia) $\left({ }^{*}\right)$ : Rh. ferrum-equinum

32.- QUATRETONDA, avenc de (Quatretonda, Valencia) $\left({ }^{*}\right)$ : $M$. schreibersi, Rh. euryale

33.- RATES I, cova de les (Torres-Torres, Valencia) $\left({ }^{*}\right)$ : Rh. ferrum-equinum, M. schreibersi, My. myotis, My. blythi, P. austriacus

34.- RATES II, cova de les (Torres-Torres, Valencia) $\left(^{*}\right)$ : $R h$. ferrum-equinum

35.- RATETES, cova de les (Corbera d'Alzira, Valencia) $\left({ }^{*}\right)$ : My. myotis, $M$. schreibersi, Rh. ferrum-equinum, My. capaccinii, My. blythi, Rh. euryale

37.- SALER, el (Valencia, Valencia) $\left(^{*}\right)$ : $P$. pipistrellus

38.- SAN ANDRÉS, túnel de (Chulilla, Valencia) $\left({ }^{*}\right)$ : Rh. ferrum-equinum (B.)

39. - SANTA, cova (Font de la Figuera, Valencia) (*): M. schreibersi (B.)

40.- SOTERRANYA, cova (Serra, Valencia) $\left({ }^{*}\right)$ : $R h$. mehelyi, Rh. euryale

41.- SUMIDORS, els (Vallada, Valencia) (*): M. schreibersi

42.- TARONGERS, masía del barranco dels (Ontinyent, Valencia) $\left(^{*}\right)$ : Rh. ferrum-equinum

43. - TAVERNES BLANQUES (Tavernes Blanques, Valencia) $\left({ }^{*}\right)$ : $P$. pipistrellus

44.- TIA ONDERA, cueva de la (Aín, Castellón) $\left(^{*}\right)$ : $R h$. hipposideros

45.- TORTERO, cueva del (Tous, Valencia) (*): $M$. schreibersi, Rh. euryale, Rh. mehelyi

46. - TOSCA, cova (Penáguila, Alicante) $\left({ }^{*}\right)$ : Rh. hipposideros, $M$. schreibersi

47.- VEDAT, urbanización del (Torrent, Valencia) $\left({ }^{*}\right)$ : T. teniotis (P.)

El listado aporta 92 citas quiropterológicas nuevas a las 110 menciones bibliográficas existentes hasta la fecha, con ello se amplía a 202 el total de referencias concretas conocidas en la Comunidad Valenciana. Por otra parte, cabe destacar la diversidad de la información aportada, pues comprende 15 especies distintas. En este sentido, el estudio da a conocer la presencia de tres especies no mencionadas con anterioridad: $R h$. mehelyi, My. daubentoni y $M y$. emarginatus. Se incrementa así el listado de murciélagos, pasando de 16 a 19 taxones. Es muy probable que dicho número aumente en cuando se realice un mayor esfuerzo prospectivo de las especies arborícolas (Nyctalus leisleri). También es importante la observación de ciertos murciélagos de los que, aunque 
ya habían sido citados, se tenía muy poca información, confirmándose así su presencia: $R$ h. hipposideros, $P$. kuhli, E. serotinus, $T$. teniotis y $P$. austriacus.

En la tabla 1 se ha resumido las distintas especies que componen la fauna quiropterológica valenciana, detallándose: el número de menciones bibliográficas, las citas aportadas por los autores, la frecuencia de cada una de las especies para el Levante español (BALCELLS, 1967 y CAROL et al., 1983) y para la Comunidad Valenciana.

\section{TABLA I}

\begin{tabular}{|c|c|c|c|c|c|}
\hline Especie & $\begin{array}{l}\mathrm{N} .^{\circ} \text { de citas } \\
\text { bibliográficas }\end{array}$ & $\begin{array}{c}\mathbf{N} .^{\circ} \text { de citas } \\
\text { nuevas }\end{array}$ & Total & $\begin{array}{c}\text { Frec. \% } \\
\text { Balcells } 1967\end{array}$ & $\begin{array}{c}\text { Frec. \% } \\
\text { actual }\end{array}$ \\
\hline Rh. ferrum-equinum & 46 & 21 & 67 & 24.1 & 33.17 \\
\hline Rh. hipposideros & 4 & 3 & 7 & 7.0 & 3.47 \\
\hline Rh. euryale & 7 & 13 & 20 & 5.2 & 9.90 \\
\hline$R h$. mehelyi & 0 & 4 & 4 & 3.4 & 1.98 \\
\hline My. emarginatus & 0 & 1 & 1 & - & 0.49 \\
\hline My. nattereri & 7 & 0 & 7 & 8.6 & 3.47 \\
\hline My. myotis & 6 & 4 & 10 & 3.5 & 4.95 \\
\hline My. blythi & 5 & 5 & 10 & 8.6 & 4.95 \\
\hline My. daubentoni & 0 & 1 & 1 & - & 0.49 \\
\hline My. capaccinii & 5 & 6 & 11 & 3.5 & 5.45 \\
\hline E. serotinus & 4 & 1 & 5 & 5.2 & 2.48 \\
\hline P. pipistrellus & 5 & 6 & 11 & 3.5 & 5.45 \\
\hline P. kuhli & & 2 & 2 & 3.5 & 0.99 \\
\hline P. savii & 1 & 0 & 1 & 3.5 & 0.49 \\
\hline B. barbastellus & 1 & 0 & 1 & 3.5 & 0.49 \\
\hline P. austriacus & 2 & 4 & 6 & 5.2 & 2.97 \\
\hline Ny. noctula & 1 & 0 & 1 & - & 0.49 \\
\hline M. schreibersi & 12 & 21 & 33 & 10.0 & 16.34 \\
\hline$T$. teniotis & 3 & 1 & 4 & 1.7 & 1.98 \\
\hline
\end{tabular}

TABLA 1.-Cuadro sinóptico de la fauna quiropterológica de la Comunidad Valenciana. Se puede observar para cada especie: el número de citas obtenidas a partir de la bibliografía, el número de menciones nuevas, la frecuencia calculada para el Levante español por BALCELLS (1967) y CAROL et al. (1983) y la frecuencia actual hallada a partir de la totalidad de citas existentes.

\section{DISCUSIÓN}

Si bien el total de especies halladas en la región valenciana es considerable, 19 taxones distintos que representan un $70.37 \%$ de las 27 especies que constituyen la fauna quiropterológica peninsular actual, la intensidad de prospección es hoy en día todavía escasa. Dicho rasgo queda reflejado en el valor de la densidad de prospección 8.67 citas $/ 1000 \mathrm{~km}^{2}$. El cálculo efectuado dista de 
la densidad teórica, propuesta por CAROL et al. (1983), para obtener un conocimiento aceptable de la fauna de un territorio de $23.291 \mathrm{~km}^{2}$, como es el valenciano. Sin embargo, si bien es cierto que existen lagunas prospectivas considerables, centradas principalmente en las provincias de Alicante y Castellón, de otras zonas se empieza a tener un notable volumen de información. Se incluiría dentro de dichas áreas, el valle del río Júcar, que presenta un importante interés quiropterológico tanto desde un punto de vista de distribución, como ecológico y etológico para las migraciones de los murciélagos. Igualmente, merece la pena destacar el aumento de citas que se ha producido en los últimos años, hecho que queda reflejado al comparar la densidad de prospección obtenida por BALCELLS (1967) para el Levante español, 1.3 citas $/ 1000 \mathrm{~km}^{2}$, con la hallada en el presente estudio para la Comunidad Valenciana, 8.67 citas $/ 1000 \mathrm{~km}^{2}$. En este sentido, al ser el Levante peninsular una área geográfica más amplia, hay que tomar los datos como indicativos. El índice de variedad de fauna da un valor de 2.19, resultado que contrasta con el hallado por BALCELLS (CAROL et al., 1983) para el Levante (12.31). Dicha disminución obedece a un fuerte incremento en el número de citas, en una región en la que ya habían sido observadas la mayor parte de las especies de murciélagos . que componen su fauna. Como ya se ha mencionado en el apartado de resultados, tal como se intuía en la nota de FAUS (1987), se han hallado tres especies todavía inéditas para la región valenciana: $R h$. mehelyi, My. emarginatus y $M y$. daubentoni (ver Fig. 2 y 6). De la primera se aportan 4 citas y ello permite sugerir que dicha especie es más frecuente de lo en principio esperado, característica que se ajusta al grupo biogeográfico en el que se la incluye, espècies mediterráneas del sur bastante estrictas (BALCELLS, 1967 y CAROL, et $a l .$, 1983). La cita de $M y$. emarginatus presenta un considerable interés al ser un quiróptero del que se posee muy poca información. En la cueva oscura se hallaron diversos ejemplares, viéndose una hembra que presentaba las glándulas mamarias muy desarrolladas. Podía tratarse de un animal en período de lactación, en cuyo caso la cita cobraría mayor interés por hacer referencia a un «Wochenstube». Una única mención se posee de My. daubentoni, quiróptero que en el sur de Francia presenta un marcado carácter fisurícola, refugiándose normalmente en cuevas durante el invierno (SAINT-GIRONS, 1973). La observación valenciana fue efectuada a mediados de mayo y en el interior de la sima de Les Graelles, donde los animales constituían un «Wochenstube». Los individuos formaban una agrupación de unas pocas decenas de ejemplares.

Por otra parte, merecen especial atención una serie de especies que aunque ya han sido mencionadas, se conocía muy poco su repartición, $P$. kuhli, $P$. austriacus, $R h$. hipposideros, $E$. serotinus y $T$. teniotis (ver Figs. 2, 4, 5 y 8). Entre ellas $P$. kuhli es una de las más significativas, pues la única mención que se poseía era la realizada por CABRERA (1914) quien atribuía la distribución a toda el área del Levante español y concretamente a Alicante. Dicha cita es muy general y no hace referencia a ninguna localidad concisa. La detección de dos enclaves concretos para la especie, es un resultado que se destaca en el actual estudio.

La especie que presenta una frecuencia más elevada es $R h$. ferrum-equinum con un $33.17 \%$, siendo el quiróptero que se conoce mejor su distribución (ver Tabla 1 y Fig. 1). Sin embargo, el resultado obtenido podría estar sobrestima- 
do por tratarse de un murciélago fácilmente visible e identificable por los espeleólogos. En este sentido, la frecuencia es probable que disminuya cuando se haya efectuado un mayor número de prospecciones exhaustivas acercándose a un valor intermedio entre el obtenido por BALCELLS (1967) y CAROL et al. (1983) y el calculado con datos actuales (ver Tabla 1). No obstante, la elevada frecuencia de $R h$. ferrum-equinum parece a primera vista, que la especie dispone de grandes efectivos en la Comunidad Valenciana, no obstante, cabe precisar el carácter poco gregario del murciélago grande de herradura en Levante, no habiéndose detectado concentraciones tan numerosas como las del SE francés, donde la especie suele formar grupos de más de cien individuos (SERRA-COBO, datos propios). El quiróptero que le sigue en frecuencia es $M$. schreibersi, constituyendo agrupaciones importantes que en la mayoría de los casos sobrepasan el centenar de ejemplares. Es obvio que una especie gregaria como $M$. schreibersi aunque le corresponda menor frecencia, tiene una mayor biomasa poblacional que $\boldsymbol{R} h$. ferrum-equinum. Cabe considerar al murciélago de cueva, como el de mayores efectivos entre las especies cavernícolas valencianas (ver Fig. 3).

También merecen algunas consideraciones las frecuencias de los murciélagos de ámbito no cavernícola, ya sean fisurícolas, arborícolas o bien de carácter antropófilo, como ocurre con $P$. pipistrellus (ver Fig. 4). Su bajo número de citas se debe a una falta de prospección adecuada, pues hasta la fecha el esfuerzo se ha concentrado en el dominio hipógeo. Es muy probable que la intensificación de campañas efectuadas fuera de las cavidades, ampliara el número de especies para la Comunidad Valenciana, obteniéndose así menciones de $N y$. leisleri, especie últimamente cada vez más observada en diversos lugares de la geografía española (BENZAL, 1984 y ARRIZABALAGA y MONTAGUD, 1984). Si la prospección de tales ámbitos se practicara, el número de citas de murciélagos no-cavernícolas aumentaría. Desde un punto de vista biogeográfico cabría agrupar los quirópteros valencianos en 5 categorías distintas (BALCELLS, 1967, NADAL et al., 1968 y CAROL et al., 1983):

1.- Especies de origen tropical y subtropical: entre las que se hallarian $P$. kuhli y $M$. schreibersi, correspondiéndoles un total de 35 citas y un $17.33 \%$.

2.- Especies mediterráneas del sur, bastante estrictas: pertenecerían a dicho grupo Rh. euryale Rh. mehelyi, My. capaccinii, My. blythi y $T$. teniotis. El número de citas es de 49 , lo que supone el $24.26 \%$.

3.- Especies mediterráneas del norte: se incluye aquí $R h$. ferrumequinum, $R$ h. hipposideros, $P$. austriacus, My. emarginatus, $M y$. myotis y $P$. savii. El número de citas hallado es de 92 y le corresponde un $45.54 \%$.

4.- Especies centro-europeas: pertenecen a dicho grupo Barbastella barbastellus, My. daubentoni y My. nattereri. El total de citas es de 8 y el tanto por ciento de 4.46 .

5.- Especies de difícil catalogación ecológica: se agrupa aquí $P$. pipistrellus, E. serotinus y $N y$. noctula, correspondiéndoles un total de 17 citas y un $8.41 \%$.

El grupo predominante es el de las especies mediterráneas del norte, ello se debe al gran número de citas existentes de $R h$. ferrum-equinum. El resultado obtenido es muy similar al hallado por BALCELLS (1967) y CAROL et al., (1983) para el Levante peninsular, 43.1\%. A continuación se encuentran las especies mediterráneas del sur, cuya frecuencia relativa es ligeramente su- 
perior a la hallada por BALCELLS (1967) y CAROL et al. (1983). Los resultados obtenidos para el grupo de quirópteros de origen tropical y subtropical, muestran un considerable incremento con respecto a los calculados para el Levante, pues se para de un $13.8 \%$ a un $17.33 \%$. Dicho aumento se debe a la aportación de nuevas citas de $M$. schreibersi. Por el contrario a las especies centro-europeas les corresponde un número relativo mucho menor (pasan del $12.1 \%$ al $4.46 \%$ ). El tanto por ciento de las especies de difícil catalogación ecológica, se mantiene igual al calculado por BALCELLS (1967) y CAROL et al. (1983). Sin embargo, si se analiza la fauna quiropterológica desde un punto de vista de abundancia, los grupos que presentan mayor biomasa son el de las especies mediterráneas del sur y las de origen tropical y subtropical. Dicha característica se ajusta perfectamente al clima mediterráneo que presenta la región.

\section{AGRADECIMIENTOS}

Los autores agradecen al Dr. Enrique Balcells la revisión desinteresada del manuscrito, así como el Dr. Guillermo Esteban y a los doctorandos Pepe Oltra y Julio Herrero del Departamento de Parasitología de la Facultad de Farmacia de Valencia y a Juan José Herrero-Borgonón, las facilidades informativas para la elaboración del presente estudio. Igual y especialmente se agradece la participación de todas aquellas personas que han intervenido de una u otra forma en las diversas campañas quiropterológicas, en forma singular a Jesús Monedero, Antonio Navarro, Nuria Mach, Ferran Pauné, Joan Francesc Guasch y Marc López; y también se menciona la colaboración de Blanca Amengual, Anna Fernández, Marta López, Liberto Mas, Xesco Mir, Josep María Sansó, Montse Sansó y Ariadna Serra. 


\section{BIBLIOGRAFÍA}

ARRIZABALAGA A. y MONTAGUD, E. 1984: Notes sobre la fauna de Quiròpters del Vallès Oriental (Barcelona, Catalunya). Una nova espècie per a la fauna espanyola. Misc. Zool., 8: 307-310, Barcelona.

BALCELLS, E. 1967: Murciélagos y nicteríbidos del Levante español. Bol. R. Soc. Española Hist. Nat., 65: 199-224, Madrid.

BAUER, K. 1956: Zur Kenntnis der Fledermausfauna Spaniens. Bonner Zool. Beitr., 7: 269-320, Bonn.

BENZAL, J. 1984: Présence de la nyctale de Leisler Nyctalus leisleri (KUHL, 1818) à Gredos (Espagne centrale). Mammalia, 48(3): 261, París.

BOSCA, E. 1915: Comentarios sobre mamíferos de la región valenciana comprendidos en la «Fauna Ibérica» de don Angel Cabrera. Mem. R. Soc. Española Hist. Nat., 10(2): 125-146, Madrid.

CABRERA, A. 1904: Ensayo monográfico sobre los quirópteros de España. Mem. R. Soc. Española Hist. Nat. (Memoria 5. a), 2: 249-287, Madrid.

- 1914: Fauna Ibérica Mamíferos. 441 págs. Museo Nacional de Ciencias Naturales, Madrid.

CAROL, A.; SAMARRA, F. J. y BALCELLS, E. 1983: Revisión faunística de los murciélagos del Pirineo Oriental y Catalunya. Monografías del Instituto de Estudios Pirenaicos, 112: 106 págs., Jaca.

DONAT, J. 1966: Catálogo Espeleológico de la provincia de Valencia. Mem. del Inst. Geol. y Min. España T. LXVII: 186 págs., Madrid.

FAUS, F. V. 1987: Contribución al conocimiento de los quirópteros de la provincia de Valencia. VIII Reunión Bieal de la R. Soc. Española His. Nat. Resúmenes de las comunicaciones, sesión paneles, pág. 68, Pamplona.

GRAELLS, M. de la P. 1987: Fauna Mastodológica Ibérica. Memoria de la Real Academia de Ciencias Exactas, Físicas y Naturales, 17: 806 págs., Madrid.

HERRERO-BORGOÑ ÓN, J. J. 1983: Introducción al conocimiento de los murciélagos cavernícolas en el País Valenciano. Lapiaz, 11: 9-14, Valencia.

HERRERO-BORGOÑÓN, J. J. y GONZÁLEZ, J. V. 1987: Aproximación a la flora y a la fauna de las cavidades subterráneas de la SAfor (Valencia). C. E. I. C. Alfonso El Viejo, Ayuntamiento de Gandía, 127 págs.

HERRERO-BORGOÑÓN, J. J. 1988: Algunas observaciones de murciélagos cavernícolas en la provincia de Castellón de la Plana. Spelaion, 4 (en prensa), Valencia.

NADAL, J.; VERICAD, J. R.; VIDAL, A.; MARTINEZ-RICA, J. P. y BALCELLS, E. 1968: Guión para trabajos prácticos. Zoologia-Cordados. Publ. Cent. pir. Biol. Exp., 350 págs., Barcelona-Jaca.

SAINT-GIRONS, M. C. 1973: Les mammifêres de France et du Benelux. Doin Editeurs, 481 págs., Paris.

SAMARRA, F. X. y CAROL, A. 1986: Murciélagos incorporados a la colección del Museo de Zoología de Barcelona durante las tres últimas décadas. Miscelánea Zoológica, 10: 305-31, Barcelona.

SERRA-COBO, J. y BALCELLS, E. 1987: Contribución a la ecología y distribución de Myotis capaccinii. Actas VIII Bienal de la R. Soc. Española Hist. Nat., 187-194, Pamplona.

SEVILLA, P. 1986: Estudio paleontológico de los quirópteros del Cuaternario español. Tesis Doctoral. Facultad de C. C. Biológicas Universidad Complutense de Madrid, 332 págs., Madrid.

VILLAPLANA, J. 1986: Introducción a la fauna vertebrada de la Safor. C. E. I. C. Alfons el Vell y Generalitat Valenciana, 213 págs., Oliva. 